\title{
THE FIFTH INTERNATIONAL CONFERENCE ON UNIFORM DISTRIBUTION THEORY (UDT 2016) \\ SOPRON, HUNGARY, JULY 5-8, 2016
}

\begin{abstract}
This volume contains papers originally presented or inspired by the Fifth International Conference on Uniform Distribution Theory which was held in Sopron, Hungary, July 5-8, 2016.
\end{abstract}

\section{Preface}

This conference is direct continuation of the 1st International Conference in Marseilles, CIRM, France, January 21-25, 2008; the 2nd International Conference in Strobl am Wolfgangsee, Austria, July 5-9, 2010; the 3rd International Conference on Uniform Distribution Theory (UDT 2012) which was held in Smolenice, Slovakia, June 25-29, 2012; the 4th International Conference on Uniform Distribution Theory (UDT 2014) Ostravice, Czech Republic, June 30-July 4, 2014.

The latest 5th International Conference on Uniform Distribution Theory was held in Sopron, Hungary, July 5-8, 2016.

The program of the conference could be characterized by the following key-words related to Probabilistic Number Theory:

Uniform distribution modulo one; Zaremba-Halton net; Legendre sequences; Thue-Morse sequence; Higher order digital sequences; $\xi(3 / 2)^{n} \bmod 1$; sequences $n \alpha \bmod 1$; Strongly u.d. sequences; Automatic sequences; Prescribed distribution; Gaussian distribution; Irregularities of distribution; Discrepancy estimates; Small $L_{p}$ discrepancy; Diaphony; Weighted sum-of-digits function; Trigonometric series; Random frequencies; lattice points; Irrational polytopes; Rational Diophantine sextuples; Spectral Banach problem; Weighted densities; On squares in finite fields; Efective diophantine results; Generalized polynomials; Minkowski-Voronoi continued fractions; Normal numbers; Nonresidue modulo a prime; 
lattice point sets; Divisible consecutive integers; Heilbronn sets; Cubic residues; Cyclic groups; linear complexity; p-adic integers; Exponential sums; Selberg zeta-function; Dedekind zeta-function; Dynamical zeta-functions; Difference set; Distribution of gaps; Consecutive gaps between primes; Digits of prime numbers; Pseudorandom binary lattices; Multiplicative subgroups; Product sets; Optimal transport; Perron numbers; Expansion complexity.

During the 5th International Conference on Uniform Distribution Theory, UDT Editorial Board meeting was held and the results are listed below.

\section{Programme committee}

Vera T. Sós, chair (Alfréd Rényi Institute of Mathematics, Budapest)

Attila Pethő (University of Debrecen, Debrecen)

András Sárközy (Eötvös Loránd University, Budapest)

\section{Organizing committee}

Krisztián Gueth (University of West Hungary, Sopron)

Tamás Herendi (University of Debrecen, Debrecen)

László Németh (University of West Hungary, Sopron)

László Szalay (University of West Hungary, Sopron)

\section{Sponsors}

Hungarien Academy of Sciences

Bolyai Society

MTA Soproni Tudós Társaság

Universitas Hungariae Occidentalis-ANNO 1735
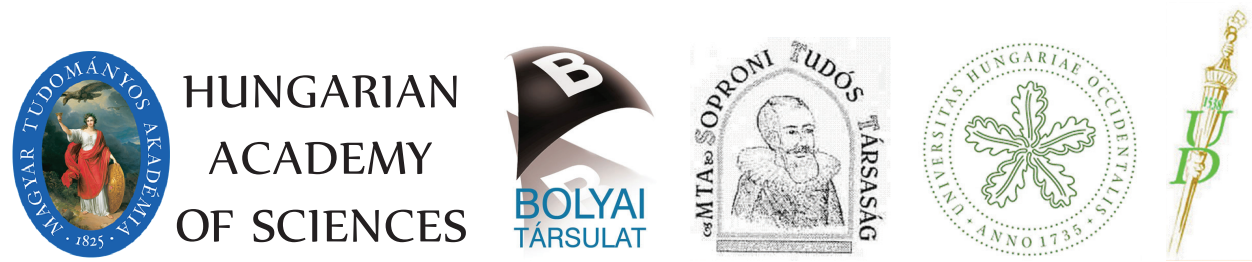

\section{Further sponsors}

Heineken Hungária Sörgyárak Zrt. Sopron

Walter Autó Kft. Sopron 
THE FIFTH INTERNATIONAL CONFERENCE ON UNIFORM DISTRIBUTION THEORY

\section{Plenary talks}

[1] Christoph Aistleitner: A cheap proof of Hinrichs' theorem

[2] Vladimír Baláž - Jana Fialová-Ladislav Mišík, Oto Strauch: Weighted sum-of-digits function

[3] ISTVÁN BERKES: Trigonometric series with random frequencies

[4] BENCE BoRDA: The number of lattice points in irrational polytopes

[5] Andrej Dujella: There are infinitely many rational Diophantine sextuples

[6] El Houcein El Abdalaoui: On the spectral Banach problem from the Scottish book and ats polynomials

[7] Ferdinánd Filip_József Bukor—JÁnos T. Tóth: The structure of weighted densities

[8] Mikhail Gabdullin: On squares in special sets in finite fields

[9] Vassil Grozdanov-Vesna Dimitrievska Ristovska: On the $\left(W_{G_{h, \phi} ; \alpha ; \beta ; \gamma}\right)$-diaphony of the net of Zaremba-Halton over finite groups (not presented)

[10] Katalin Gyarmati: On the complexity of a family of Legendre sequences with irreducible polynomials

[11] KÁLmÁn GYÖRY: General efective diophantine results over finitely generated domains (not presented)

[12] JÁnos Folláth-TAmás HeREnd: On construction of sequences with Gaussian distribution

[13] Roswitha Hofer: Uniform distribution modulo one of generalized polynomials

[14] Gyula Károlyi: Irregularities of distribution with respect to round convex regions in the plane

[15] Oleg Karpenkov: Combinatorics of Minkowski-Voronoi multidimensional continued fractions

[16] ImRE KÁtAI: Strongly UD mod 1 sequences, strongly q-ary normal numbers

[17] Sergei Konyagin: The Smallest simultaneous power nonresidue modulo a prime 
THE FIFTH INTERNATIONAL CONFERENCE ON UNIFORM DISTRIBUTION THEORY

[18] RALPh KRITZINGeR: Symmetrized point sets in the unit square with small $L_{p}$ discrepancy

[19] HeLENE LAIMER: Reduced fast component-by-component constructions of lattice point sets

[20] Gerhard Larcher: On discrepancy estimates for sequences

[21] FlORIAN LUCA: Consecutive integers divisible by the number of their divisors

[22] Manfred G. Madritsch: Multidimensional Heilbronn sets

[23] Máté Matolcsi: Sets avoiding quadratic or cubic residues in cyclic groups

[24] Christian Mauduit: The subsequence of Thue-Morse sequence along squares is normal

[25] LÁSZLÓ MÉRAi: On the linear complexity of automatic sequences

[26] LAdislav MišÍK-JÁnos T. Tóth: Subsequences with prescribed distribution

[27] RADHAKRISHNAN NAIR: On distribution of subsequences of the natural numbers on the p-adic integers

[28] Werner GEORG NowAK: Estimates for single exponential sums

[29] Friedrich Pillichshammer: $L_{p}$-discrepancy and beyond of higher order digital sequences

[30] JÁnos PINTZ: Some problems of Erdős about consecutive gaps between primes

[31] Štefan PorubskÝ: A geometric approach to properties of sequences no

[32] Sudhir Pujahari: Distribution of gaps between equidistributed sequences

[33] Jö̈L RIVAT: On the digits of prime numbers

[34] ImRe Ruzsa: Differences and multiple sums

[35] RichÁRD SEBÖK: On imbalanced pseudorandom binary lattices

[36] Ilya ShKREDOV: On additive decomposition of multiplicative subgroups

[37] IURII ShteInikov: On the product sets of rational numbers

[38] RAIVYDAS ŠImÉNAS: On the distribution of the a-values of the Selberg zeta-function

[39] Ото Strauch: Mahler's conjecture on $\xi(3 / 2)^{n} \bmod 1$

[40] RoBERT TICHY: Uniform distribution and optimal transport 
THE FIFTH INTERNATIONAL CONFERENCE ON UNIFORM DISTRIBUTION THEORY

[41] Artem Uvakin: About two-dimensional sums' generalization

[42] JeAn-Louis Verger-Gaugry: Dynamical zeta functions, asymptotic expansions and Perron numbers from trinomials

[43] Arne Winterhof: Expansion complexity and linear complexity of sequences over finite fields

\section{Results of UDT Editorial Board meeting}

- There were the new members of the Editorial Board named:

Peter Grabner, Katalin Gyarmati, Peter Kritzer, Gerhad Larcher, János Pintz, Jean-Luis Verger-Gaugry.

- There was appointed by members of Conference Commitee, namely by Oleg Kapenkov, Jean-Louis Verger Gaugry and Radhakrishnan Nair, that the upcoming conference UDT 2018 will be held at CIRM, at Luminy, France, on October 1-5, 2018.

\section{List of participants}

1. Christoph Aistleitner (TU Graz), aistleitner@math.tugraz.at

2. Vladimír Baláž (Slovak Technical University), vladimr.balaz@stuba.sk

3. István Berkes (TU Graz), berkes@tugraz.at

4. András Biró (MTA Rényi Intézet), biroand@renyi.hu

5. József Borbély (University of Obuda), borbely.jozsef@amk.uni-obuda.hu

6. Bence Borda (Rutgers University, New Jersey), bordabence85@gmail.com

7. Jean-Marie De Koninck (Laval University), jmdk@mat.ulaval.ca

8. Andrej Dujella (University of Zagreb), duje@math.hr

9. El Houcein El Abdalaoui (University of Rouen Normandy), elabdelh@gmail.com

10. Ferdinánd Filip (J. Selye University), filipf@ujs.sk

11. Mikhail Gabdullin (MUS), gabdullin.mikhail@ya.ru,

12. Vassil Grozdanov (South West University Neofit Rilski), vassgrozdanov@yahoo.com (absent)

13. Krisztián Gueth (University of West-Hungary), guethk@gmail.com

14. Katalin Gyarmati (Eötvös Loránd University), gykati@cs.elte.hu 
15. Kálmán Győry (University of Debrecen), gyory@science.unideb.hu (absent)

16. Peter Hellekalek (University of Salzburg), peter.hellekalek@sbg.ac.at

17. Tamás Herendi (University of Debrecen), herendi@inf.unideb.hu

18. Roswitha Hofer (Johannes Kepler University Linz), roswitha.hofer@jku.at

19. Gyula Károlyi (Alfréd Rényi Institute of Math.), karolyi@cs.elte.hu

20. Oleg Karpenkov (University of Liverpool), karpenk@liv.ac.uk

21. Imre Kátai (ELTE), katai@inf.elte.hu

22. Sergei Konyagin (Steklov Institute of Mathematics), konyagin23@gmail.com

23. Ralph Kritzinger (Johannes Kepler University Linz), ralph.kritzinger@jku.at

24. Manfred Kühleitner (BOKU Vienna), manfred.kuehleitner@boku.ac.at

25. Helene Laimer (Ricam Linz), helene.laimer@ricam.oeaw.ac.at

26. Gerhard Larcher (University Linz), Gerhard.Larcher@jku.at

27. Florian Luca (Wits University), florian.luca@wits.ac.za

28. Manfred Madritsch (Université de Lorraine), manfred.madritsch@univ-lorraine.fr

29. Máté Matolcsi (Rényi Institute, Budapest), matemato@gmail.com

30. Christian Mauduit (Université d'Aix-Marseille), mauduit@iml.univ-mrs.fr

31. László Mérai (RICAM), merai@cs.elte.hu

32. Ladislav Mišík (Universty of Ostrava), ladislav.misik@osu.cz

33. Radhakrishnan Nair (The University of Liverpool), nair@liv.ac.uk

34. László Németh (University of West Hungary), nemeth.laszlo@emk.nyme.hu

35. Mario Neumüller (JKU Linz), mario.neumueller@jku.at

36. Werner Georg Nowak (BOKU Vienna,) nowak@boku.ac.at

37. Attila Pethő (University of Debrecen), petho.attila@inf.unideb.hu

38. Friedrich Pillichshammer (Johannes Kepler University Linz), friedrich.pillichshammer@jku.at 
39. János Pintz (Rényi Institute, Hungarian Academy of Sciences), pintz.janos@renyi.mta.hu

40. Štefan Porubský (Institute of Computer Science, Prague), sporubsky@hotmail.com

41. Florian Puchhammer (Johannes Kepler University Linz), florian.puchhammer@jku.at

42. Joël Rivat (Universit d'Aix-Marseille), joel.rivat@univ-amu.fr

43. Imre Ruzsa (Rényi Institute), ruzsa@renyi.hu

44. András Sárközy (Eötvös Loránd University), sarkozy@cs.elte.hu

45. Richárd Sebők (Eötvös Loránd University), sebokr@cs.elte.hu

46. Ilya Shkredov (Steklov Institute of Mathematics), ilya.shkredov@gmail.com

47. Iurii Shteinikov (Steklov Institute of Mathematics), yuriisht@gmail.com

48. Raivydas Šiménas (Vilnius University), raivydas.simenas@mif.vu.It

49. Vera T. Sós (Rényi. Math. Inst. of Hungarian Acad. of Sciences), sos@renyi.hu

50. Oto Strauch (Mathematical Institute of the Slovak Academy of Siences), oto.strauch@mat.savba.sk

51. László Szalay (University of West Hungary), szalay.laszlo@nyme.hu.

52. Mihály Szalay (Eötvös Loránd University), mszalay@cs.elte.hu

53. Szabolcs Tengely (University of Debrecen), tengely@science.unideb.hu

54. Robert Tichy (TU Graz), tichy@tugraz.at

55. Artem Uvakin (Moscow State University), artemuvakin@gmail.com

56. Jean-Louis Verger-Gaugry (CNRS University Savoie Mont Blanc), Jean-Louis. Verger-Gaugry@univ-smb.fr

57. Arne Winterhof (RICAM), arne.winterhof@oeaw.ac.at 